\title{
Help, I am going to have ERCP should i be worried about the endoscope.....or the anesthetic?
}

\author{
Volume 2 Issue 6 - 2015 \\ Gerald Bushman \\ Anesthesia Critical Care Medicine, United States

\begin{abstract}
Correspondence: Gerald Bushman, Pediatric and Cardiac School of Medicine, Children $\square$ s Hospital Los Angeles, 4650 Sunset Blvd., Los Angeles CA 90027, Tel (323) 36I-2262, Email gbushman@chla.usc.edu
\end{abstract} \\ Anesthesiology, Anesthesia Critical Care Medicine, Keck USC
}

\section{Opinion}

Innovations in procedural medical care often become standard and frequent treatment after a certain amount of medical literature exists extolling the benefits of the procedure, regulatory agencies indicate no objection based on a low likelihood of serious complications, and the technology and knowledge basis required for the innovation reach a large number of practitioners. In the United States (US), Endoscopic Retrograde Cholangiopancreatography (ERCP), a procedure that uses duodenoscopic endoscopy and fluoroscopic imaging to diagnose and treat diseases of the biliary or pancreatic ducts, has recently come under criticism for outbreaks of post-procedural sepsis involving carbapenem-resistant Enterobacteriaceae (CRE), a highly lethal bacteria which has highly evolved antibiotic resistance. In a major medical center in Los Angeles, nearly 200 exposures have been identified, at least two fatalities noted, and others are being closely monitored in-hospital for infection after ERCP performed with the prototypical endoscope used for the procedure (Olympus). ${ }^{1}$ Since 2012, similar outbreaks have been seen and reported in the lay press, most involving medical centers that provide the ERCP procedure on a high-volume basis. Up to 500,000 ERCP procedures are performed in the US on an annual basis. The difficulty seems to be that despite US Food and Drug Administration sanctioned procedures for sterilization of the device after an ERCP, there is a small "elevator" channel in the scope used for instruments and directing the scope that is not adequately accessed by sterilizing agents during the approved decontamination procedures.

Clusters of serious complications such as these typically attract the attention of the US Centers for Disease Control, Food and Drug Administration, local departments of Public Health, and other federal and state resources to provide expertise in studying the outbreak, and making recommendations and sometimes regulatory law in order to safeguard the public trust.

Although serious, the recent increase in post procedure infectious complications associated with a medical device is not the only blemish on the record of ERCP in the last decade. It has not attracted the attention of the press or regulatory agencies, but anesthetic complications associated with the ERCP procedure occur at an alarmingly high rate and with occasional catastrophic outcome. Unfortunately, in the US, when a medical device is the culprit in serious complications, there are well-established protocols for investigation and study, and remedies are well vetted and studied to assure their effectiveness. In contrast, when complications are attributed to a common management error by physicians, it is much more problematic to study and correct. It is not uncommon in the US for the civil legal system to be involved in apportioning liability for patient injury based on the legal considerations of standard of care (and whether it was breached by the practitioner), whether the adverse outcome is related to the breach in
Received: February 28, 2015 | Published: August 21, 2015

the standard of care, and how damages should be compensated to the patient by the parties deemed to be liable.

ERCP procedures are increasingly performed on an aging population for both acute and chronic conditions. Patients are routinely positioned prone for the procedure in a somewhat darkened room. Duodenoscopy is performed and fluoroscopy imaging utilized to diagnose and endoscopically treat problems in the pancreatobiliary system. Depending on the skill and experience of the gastroenterologist, the actual procedure performed, and the patient's condition, the procedure may be relatively brief or may be somewhat lengthy. The length of the procedure is not reliably predictable beforehand.

As influenced by institutional protocols and other considerations, many of these procedures are performed under some level of sedation or Monitored Anesthesia Care (MAC) and others are performed under general anesthesia with endotracheal intubation (GETA). I am frequently involved as an expert witness for either plaintiffs (injured patients) or defendants (physicians) involved in medicolegal proceedings, and I have reviewed at least several such procedures that resulted in patient harm in the past decade. The "mishaps" typically occur in the context of a MAC without endotracheal intubation. There is often commonality of the clinical circumstances, and the plaintiff and defense expert declarations regarding the event. A significant monetary award and a judgment or settlement on behalf of the plaintiff (patient) or his estate is common.

I am convinced that the clinical situation of providing MAC for ERCP is very difficult for the anesthesiologist. It is known that ASA 3 or higher patients undergoing sedation have more cardiorespiratory events during the provision of MAC and require more airway "rescues" ${ }^{2,3}$ The anesthesia provider's ability to recognize significant airway embarrassment despite the use of recommended monitors as recommended by the American Society of Anesthesiologists, including capnography (ETCO2) and pulse oximetry is seriously 
compromised by the prone position, darkened room, fluoroscopy equipment, endoscope in the mouth without a device guarding the airway, and the anxious enthusiasm of the gastroenterologist to complete the procedure. The eventual rescue of the airway in a patient who is not adequately breathing may not be timely enough to prevent a disastrous outcome. Interestingly, although the frequency of cardiorespiratory events is highest in medically fragile patients, the most disastrous outcomes may occur in otherwise healthy patients who are thought by the anesthesiologist to be unlikely to have a complication. In "selecting" a patient as suitable for MAC for ERCP, in the preanesthetic assessment the anesthesiologist typically relates a "normal" airway exam and a physical status consistent with and no comorbidities that contraindicate the planned sedation technique without intubation in the prone position. The defense of the technique often refers to the "community standard" that many ERCPs are done with MAC, and that it is the preference of the gastroenterologist that the patient not be intubated.

Unfortunately for the anesthesiologist (defendant), the legal "standard of care" issue they are found to have failed to provide is not simply the choice of the anesthetic technique often advocated by the gastroenterologist, but the failure to diagnose and treat a compromised airway under sedation/anesthesia. The initial argument by the plaintiff's (injured patient's) expert may be the arguable point of what the standard of care in the choice of anesthesia technique is and who should make that choice "in the community", gastroenterologist or anesthesiologist. There is some literature anecdotally presenting widespread use of MAC in some communities. But then the plaintiff's expert focuses on the expectation that a trained anesthesia provider should be able in a monitored procedural environment to diagnose and intervene when a patient suffers important respiratory compromise from the procedure (including the position) or the anesthetic technique, and the expected outcome of those events in a patient with a "normal airway" and no important comorbidities should not be neurologic injury or death. The anesthesiologist ironically weakens his own defense in his assertion in the preanesthesia evaluation that the patient should tolerate the procedure, because otherwise, he would choose general anesthesia with intubation. The gastroenterologist is not typically liable in such cases (unless he impedes the anesthesiologist from more expedient airway rescue such as insistence on just a bit more time to finish the procedure.)

The literature on the issue is interesting. There is literature describing that MAC is a common approach to the anesthetic care of these patients. My impression is that despite such practice in some hospitals, few anesthesiologists are comfortable with this approach. We are aware of the changes in respiratory mechanics such as functional residual capacity, closing volume and capacity, and work of breathing when patients are in the prone position, especially if they are obese. I believe most anesthesiologists would never perform a general anesthetic for even a brief prone procedure in other surgical cases without securing the airway.

Setting aside the "anecdotal" literature "surveying" the safety of practices for ERCP, what do we actually know and what would a plaintiff's expert confront us with if we were to be sued over an adverse event? ERCP is more likely to fail with MAC compared to general anesthesia., ${ }^{4,5}$ In fact, the only important endoscopy complication reported with significant frequency is the failure to complete the procedure. All of the other procedural complications are characterized as anesthesia mishaps. The frequency of the recently noted iatrogenic postprocedural infections is still very low compared to anesthesia complications.
Deep sedation can easily progress unintended to a level of anesthesia associated with respiratory compromise, and ETCO2 monitoring in the patient who has an uninstrumented airway is subject to artifact and occasionally difficult to objectively interpret. The prone position is a significant barrier to efficient rescue of the airway when a patient's own protective reflexes are abolished and respiratory mechanics are impaired by the scope, position, or airway obstruction.

Aspiration under MAC is unusual, however it is increased in procedures that are more difficult or prolonged, a category that cannot be reliably predicted beforehand. ${ }^{6} \mathrm{~A}$ critical incident rate of $9 \%$ and a mortality rate of $2 \%$ of ERCP under MAC have been quoted by in the literature pertaining to a study with a large number of ASA III or above patients. ${ }^{7}$ I believe it is likely that in the last decade of increases in the performance of ERCP that adverse events have been increasingly unreported because of medicolegal considerations. In a similar review of less ill patients, a procedure mortality rate of $0.4 \%$ was still noted under MAC. ${ }^{8}$ The most common cause of procedure related morbidity or mortality is anesthesia related. ${ }^{7}$ Compared to contemporary anesthesia mortality rates, even this lower rate is very high. If the anesthesia mortality rate overall in a operating suite or the complication rate of sedation/anesthesia services in a pediatric radiology suite approached this rate, there would be a serious need for quality improvement activity to understand the problems. If one thinks critically about what that mortality rate means, it is obvious that appropriately trained providers who have a remarkable safety record regarding managing complications of MAC under different conditions are less successful in managing those same complications under the conditions of an ERCP. To not recognize that failure as per se evidence that ERCP conditions are substantively different from those other clinical situations where MAC is more safely accomplished is a serious cognitive error. The ERCP procedural conditions are inherently a risk factor for anesthesia care under MAC.

Even in the absence of mortality, important hypoxemia occurs in $13 \%$ of patients and airway maneuvers are required in $14 \% .{ }^{3}$ Although MAC techniques including propofol infusions are associated with desirable recovery profiles compared to boluses of narcotics and tranquilizers, ${ }^{9,10}$ increasingly the patients presenting for ERCP are older, in poor health, or obese. Although propofol may be used in these patients, significant numbers will have airway or hemodynamic issues requiring attention. Referring to the package insert for propofol, I am impressed at how vigorously the manufacturer discourages the use of propofol for debilitated patients.

Although some may argue that careful patient assessment allows the selective choice of who undergoes MAC and who has a GETA, literature supporting that argument is lacking and it is a seriously flawed argument to make to defend your failure to rescue an airway in a patient who has an adverse outcome. The incorrect assessment of the patient's risk is not the deviation from the standard of care, however the failure to diagnose respiratory difficulty and rescue the airway is.

For this author, I am confident the FDA and CDC will be successful in helping Olympus resolve their disinfection protocols. But if I present for an ERCP and you are my anesthesiologist, I will be anesthetized and intubated for the procedure.

\section{Acknowledgements}

None.

\section{Conflicts of interest}

The authors declare that there are no conflicts of interest. 


\section{Funding}

None.

\section{References}

1. Terhune C. Superbug Linked to 2 Deaths at UCLA Hospital; 179 Potentially Exposed. Los Angeles Times. 2015.

2. Williams EJ, Taylor S, Fairclough P, et al. Are we meeting the standards set for endoscopy? Results of a large- scale prospective survey of endoscopic retrograde cholangio- pancreatograph practice. Gut. 2007;56(6):821-829.

3. Coté GA, Hovis RM, Ansstas MA, et al. Incidence of sedationrelated complications with propofol use during advanced endoscopic procedures. Clin Gastroenterol Hepatol. 2010;8(2):137-142.

4. Laurie T, Chang GL, Hammerman S, et al. Comparison of Anesthesia Associated (AA) sedation with Conscious Sedation (CS) in successful completion of Endoscopic Retrograde Cholangiopancreatography (ERCP)". Gastrointestinal Endoscopy. 2005;61(5):AB118.
5. Penston J, Southern P, Penston V, et al. ERCP in a district general hospital in England:a review of 1550 procedures over nine years. The Internet Journal of Gastroenterology. 2009;8(1).

6. ANZCA statement, "Anaesthesia/sedation for endoscopy. Beware unexpected aspiration".

7. M Cullinane, AJ Gray. Scoping our practice. NCEPOD 2004 Report of the National Enquiry into patient outcome and death. 2004.

8. The joint Royal Colleges of Anaesthetists (RCoA) and British Society of Gastroenterology (BSG) Working Party."Guidance for the use of propofol sedation for adult patients undergoing Endoscopic Retrograde Cholangiopancreatography (ERCP) Diagnostic and Therapeutic Endoscopy and other complex upper GI endoscopic procedures". 2011.

9. Riphaus N, Stergiou, T Wehrmann."Sedation with propofol for routine ERCP in high-risk octogenarians: a randomized, controlled study". Am J Gastroenterol. 2005;100(9):1957-1963.

10. Vargo JJ, Cohen LB, Rex DK, et al. "Nonanesthesiologist administration of propofol for GI endoscopy". Gastrointest Endosc. 2009;70(6):10531059. 